\section{Tendências da mortalidade por \\ causas externas, em São Luís, MA, de 1980 a 1999}

\section{Trends in mortality due to external causes in São Luis, MA, Brazil, from 1980 to 1999}

José Ribamar Moraes

Departamento de Patologia

Faculdade de Medicina

Universidade Federal do Maranhão (UFMA)

Praça Madre Deus, 2 - Madre Deus

65025-560 - São Luís - MA

ribamarmoraes@uol.com.br

\section{Antônio Augusto Moura da Silva}

Departamento de Saúde Pública

Faculdade de Medicina

Universidade Federal do Maranhão (UFMA)

\section{Fernando Lamy Filho}

Departamento de Medicina III

Faculdade de Medicina

Universidade Federal do Maranhão (UFMA)

\section{Raimundo Antônio da Silva}

Departamento de Saúde Pública

Faculdade de Medicina

Universidade Federal do Maranhão (UFMA)

\section{Resumo}

A mortalidade por causas externas ocupa atualmente o segundo lugar entre todas as causas de óbito no Brasil. Poucos estudos têm sido realizados a este respeito no Nordeste do Brasil, no Maranhão em especial, Estado com um dos menores coeficientes de mortalidade por estas causas. Este trabalho teve por finalidade analisar a tendência da mortalidade por causas externas em São Luís, Maranhão, de 1980 a 1999, e verificar se esta tendência difere daquela observada para o país como um todo e para outros municípios brasileiros. As causas foram analisadas de acordo com as categorias da Classificação Internacional de Doenças (CID), 9a Revisão, de 1980 a 1995, e 10ª Revisão, de 1996 a 1999. Codificação especial foi utilizada para assegurar comparabilidade entre as duas revisões da CID. Para a análise de tendência, os dados foram ajustados pelo modelo de regressão de Poisson. Houve uma tendência de queda da mortalidade altamente significante por causas externas em geral e em ambos os sexos. A mortalidade também se reduziu nas faixas etárias de 0 a 9, 30 a 39 e 40 a 49 anos de idade. Os acidentes de transporte, demais acidentes e "ignorados" apresentaram tendência de queda significante. Entretanto, os homicídios e os suicídios apresentaram tendência de aumento altamente significante. A tendência de redução da mortalidade por causas externas está de acordo com observações recentes de decréscimo para o Brasil como um todo, para a região das Américas e para o município de São Paulo.

Palavras-chave: Causas externas. Mortalidade. Violência. Homicídio. Acidentes de transporte. Análise de séries temporais. 
Abstract

Mortality due to external causes is the second leading cause of death in Brazil. Few studies addressing this issue have been conducted in the Northeast of the country, or in Maranhão, state whose mortality rate due to external causes is one of the lowest in Brazil. This paper aims at examining trends in mortality due to external causes in São Luís, from 1980 to 1999 and verifying if this trend differs for those observed for the country as a whole and for other Brazilian cities. The external causes were surveyed in accordance to the $9^{\text {th }}$ revision of the International Classification of Diseases (ICD) from 1980 to 1995 and the $10^{\text {th }}$ revision was used from 1996 to 1999. Special coding was used to assure comparability between the two ICD revisions. Trend analysis was performed using a Poisson regression model. Mortality by external causes showed a significant overall reduction, for both sexes, and in the 0-9, 30-39 and 40-49 age groups. Mortality by transportation accidents, other accidents and unknown causes reduced significantly, whereas mortality by homicides and suicides showed a highly significant increase. This decreasing trend in mortality due to external causes is in agreement with recent observations made for the country as a whole, for the Americas and for the city of São Paulo.

Keywords: External causes. Mortality. Violence. Homicides. Transport accidents. Time series analysis.

\section{Introdução}

As causas externas são a segunda causa de mortalidade no Brasil e a primeira causa de mortalidade dos 5 aos 49 anos. De 1977 a 1994 , aumentou $40 \%$, tendo o aumento sido mais elevado no sexo masculino $(47,2 \%)$ do que no sexo feminino $(15,7 \%)^{1}$. O coeficiente de mortalidade por causas externas esteve em ascensão até 1996, quando atingiu 75,9 óbitos por 100.000 habitantes, tendo declinado para 71,3 por 100.000 habitantes em 1999. O coeficiente de mortalidade por causas externas em 1999 foi mais elevado nas regiões Sudeste (87,3 por 100.000 hab) e Centro-Oeste (80,2 por 100.000 hab), apresentando valor intermediário para a região Sul (66,7 por $100.000 \mathrm{hab}$ ) e sendo mais baixo nas regiões Norte (51,3 por 100.000 hab) e Nordeste (52,7 por 100.000 hab). Roraima foi o Estado que apresentou o maior coeficiente, 148,7 por 100.000 habitantes, enquanto o Maranhão apresentou o menor, 24,7 por 100.000 habitantes ${ }^{2}$. Na região das Américas, o coeficiente de mortalidade por causas externas declinou de 1980 a meados da década de 90 em quase todos os países ${ }^{3}$.

No Brasil, entre as causas externas, a mortalidade por acidentes de transporte ocupou o primeiro lugar até o início da década de 90, sendo ultrapassado a partir daí pelos homicí$\operatorname{dios}^{4,5}$. A mortalidade por acidentes de transporte vem declinando recentemente, tendo passado de 29,8\% em 1996 para 25,3\% em 1999. Este declínio pode ser atribuído a vários fatores, dentre os quais menor velocidade, maior fiscalização, implantação de lombadas eletrônicas, maior uso de cinto de segurança, aumento na frota de carros, o que pode levar à redução da velocidade dos veículos, entre outros $^{2}$. Entretanto, a mortalidade por homicídios continua em franca ascensão $0^{6-8}$, tendo sido observado leve declínio na mortalidade por suicídios e demais acidentes ${ }^{2}$. O aumento da mortalidade por homicídios tem sido atribuído a mudanças marcantes ocorridas no Brasil, tais como o aumento das desigualdades entre as classes sociais, do número de pobres e de miseráveis, da concentração da riqueza e o incremento do tráfico de drogas e 
da criminalidade em algumas cidades brasileiras $^{6,7,9}$.

A tendência da mortalidade por causas externas apresenta grande variação entre as regiões e capitais brasileiras, por motivos pouco estudados e conhecidos ${ }^{1}$. Poucos estudos têm sido realizados no Nordeste do Brasil a este respeito e poucos trabalhos têm analisado a tendência da mortalidade por causas externas em séries temporais que incluam ao mesmo tempo os anos em que a nona e a décima revisão da CID (Classificação Internacional de Doenças) foram utilizadas.

No presente trabalho é proposta uma codificação que busca assegurar comparabilidade entre a nona e a décima revisões da CID para a análise de tendências da mortalidade por causas externas. Teve como objetivo analisar a tendência da mortalidade por causas externas, de forma geral, segundo sexo, idade e tipo de causa em uma capital do Nordeste brasileiro, São Luís, Maranhão, de 1980 a 1999, que teve, em 1994, o terceiro menor coeficiente de mortalidade por causas externas entre as capitais brasileiras ${ }^{1}$. Verificou-se se a tendência da mortalidade por causas externas, em local onde a magnitude do problema é menor, São Luís, diferiu da observada para o país como um todo, para outros municípios brasileiros e para a região das Américas.

\section{Material e métodos}

A série histórica estudada baseou-se em dados oficiais e secundários do Sistema de Informação sobre Mortalidade (SIM), do Ministério da Saúde, publicados por essa mesma instituição. Foram efetuadas análises descritivas da mortalidade proporcional por causas externas, dos coeficientes de mortalidade por causas externas, geral e específicos segundo sexo, grupo etário e tipo de causa. Para os cálculos dos coeficientes foram utilizados os dados da população residente em São Luís, no período de 1980 a 1999, obtidos na página do DATASUS na Internet ${ }^{10}$.

As causas externas foram consideradas de acordo com a $9^{\mathrm{a}}$ revisão da Classificação Internacional de Doenças (CID), códigos E800 a $\mathrm{E} 999^{11}$, de 1980 a 1995. De 1996 a 1999 foi utilizada a 10 $0^{\mathrm{a}}$ revisão da CID, códigos V01 a $\mathrm{Y}^{12}{ }^{12}$. Para assegurar comparabilidade entre as duas revisões, compararam-se as duas classificações, verificando-se os itens de mesmo significado e estabelecendo-se a equivalência entre ambas. A idéia básica foi usar como modelo a $10^{\mathrm{a}}$ revisão da CID e agrupar os itens da $9^{\text {a }}$ revisão a partir da nova classificação. Foram utilizadas 5 categorias: acidentes de transporte, demais acidentes, suicídios, homicídios e ignorados. Os códigos E800 a E848 da 9a revisão foram considerados correspondentes a acidentes de transporte, códigos V01 a V99 da 10 a revisão, englobando os de trânsito de veículos a motor (E810 a E819) e itens que na $9^{a}$ revisão pertenciam aos demais acidentes, mas que na $10^{\mathrm{a}}$ revisão passaram a fazer parte dos acidentes de transporte (E800 a E807 e E820 a E848). Como demais acidentes foram considerados os códigos E850 a E949 na 9a revisão e os códigos W00 a W99, X00 a X59 e Y40 a Y99 na décima revisão. Os suicídios e lesões auto-infligidas na $9^{\mathrm{a}}$ revisão, códigos E950 a E959, foram considerados equivalentes às lesões autoprovocadas voluntariamente na $10^{\mathrm{a}}$ revisão, códigos X60 a X84. Os homicídios e lesões provocados intencionalmente por outras pessoas, intervenções legais e operações de guerra na $9^{\mathrm{a}}$ revisão, códigos E960 a E969, E970 a E978 e E990 a E999 foram considerados equivalentes às agressões, intervenções legais e operações de guerra na $10^{a}$ revisão, códigos X85-X99, Y00Y09 e Y35-Y36. Os códigos E980-E989 na 9a revisão, lesões em que se ignora se foram acidental ou intencionalmente infligidas, foram consideradas correspondentes aos eventos (fatos) cuja intenção é indeterminada na $10^{\mathrm{a}}$ revisão, códigos Y10 a Y34.

Para a análise de tendência, os dados correspondentes à série temporal de $1980 \mathrm{a}$ 1999 foram ajustados por modelos de regressão de Poisson. Os dados de óbitos e as estimativas populacionais não são sujeitas ao erro amostral, pois compreendem a totalidade dos eventos ocorridos. Entretanto, sofrem os efeitos do erro aleatório no processo de registro, contagem populacional e estimação dos valores intercensitários. Por isso 
é necessário adotar métodos para se lidar com o erro aleatório na comparação de coeficientes ao longo do tempo. A regressão de Poisson tem sido um método utilizado para este fim, pois é adequada para eventos raros como os aqui estudados ${ }^{13}$. Adotou-se o nível de significância de 0,05 para se verificar a probabilidade de o acaso explicar se a variação do risco relativo foi significante ou não. A bondade do ajuste dos dados ao modelo foi verificada pelo teste do qui-quadrado, comparando-se os dados observados com os preditos pelo modelo. Foram utilizados os programas Tabwin e Stata ${ }^{14}$ na tabulação e análise estatística.

\section{Resultados}

De 1980 a 1999, as mortes por causas externas fizeram em São Luís 8.769 vítimas, o que representou aproximadamente $12 \%$ do total dos óbitos ocorridos no período.
Proporcionalmente, no início da série, dentre as causas externas predominava a mortalidade por acidentes de transporte e demais acidentes. No final da série, excluindose os ignorados, os demais acidentes juntamente com os homicídios passaram a ser as mais freqüentes causas de óbito entre as causas externas (Tabela 1).

Os maiores coeficientes de mortalidade por causas externas foram verificados nos anos de 1982 e 1988, com 83,4 por 100.000 habitantes e 79,1 por 100.000 habitantes, respectivamente. A partir de então apresentaram ligeiro declínio até atingir o seu valor mais baixo em 1994 com 52,9 por 100.000 habitantes, encerrando o período com 53,4 por 100.000 habitantes, valor inferior ao inicial que foi de 63,9 por 100.000 habitantes em 1980 (Tabela 2). Apesar das flutuações, observou-se tendência decrescente da mortalidade por causas externas, com redução de $1,4 \%$ ao ano (Tabela 3 ).

Tabela 1 - Número de óbitos por causas externas e distribuição percentual segundo tipo de causa em São Luís, 1980 a 1999.

Table 1 - Number of deaths due to external causes and percentage distribution according to the type of cause in São Luís, 1980 to 1999

\begin{tabular}{lcccccc}
\hline Ano & $\begin{array}{c}\text { Causas } \\
\text { Externas } \\
\mathrm{n}\end{array}$ & $\begin{array}{c}\text { Acidentes de } \\
\text { Transporte } \\
\%\end{array}$ & $\begin{array}{c}\text { Demais } \\
\text { acidentes } \\
\%\end{array}$ & $\begin{array}{c}\text { Suicídios } \\
\%\end{array}$ & $\begin{array}{c}\text { Homicídios } \\
\%\end{array}$ & $\begin{array}{c}\text { Ignorados } \\
\%\end{array}$ \\
\hline 1980 & 287 & 39,7 & 12,9 & 3,5 & 16,0 & 27,9 \\
1981 & 366 & 39,3 & 36,9 & 3,0 & 19,7 & 1,1 \\
1982 & 410 & 22,4 & 54,9 & 0,0 & 12,9 & 9,8 \\
1983 & 404 & 11,4 & 52,2 & 1,7 & 3,7 & 30,9 \\
1984 & 374 & 11,0 & 51,9 & 1,3 & 6,1 & 29,7 \\
1985 & 395 & 10,1 & 60,0 & 1,0 & 6,3 & 22,5 \\
1986 & 442 & 19,0 & 47,3 & 2,7 & 15,6 & 15,4 \\
1987 & 434 & 27,4 & 39,6 & 1,8 & 21,0 & 10,1 \\
1988 & 498 & 33,5 & 35,7 & 3,0 & 23,7 & 4,0 \\
1989 & 453 & 34,7 & 26,7 & 2,0 & 35,5 & 1,1 \\
1990 & 450 & 32,4 & 27,8 & 2,7 & 35,8 & 1,3 \\
1991 & 478 & 31,2 & 24,5 & 4,6 & 38,7 & 1,0 \\
1992 & 424 & 37,0 & 22,2 & 2,8 & 36,8 & 1,2 \\
1993 & 424 & 31,8 & 29,2 & 4,2 & 30,0 & 4,7 \\
1994 & 396 & 31,6 & 25,8 & 3,8 & 35,1 & 3,8 \\
1995 & 508 & 28,9 & 28,0 & 3,3 & 36,2 & 3,5 \\
1996 & 542 & 25,3 & 31,2 & 5,9 & 30,6 & 7,0 \\
1997 & 524 & 19,7 & 30,9 & 4,8 & 30,5 & 14,1 \\
1998 & 513 & 12,7 & 24,2 & 3,7 & 24,0 & 35,5 \\
1999 & 447 & 17,7 & 30,9 & 5,4 & 22,4 & 23,7 \\
\hline
\end{tabular}


Tabela 2 - Número de óbitos e coeficientes de mortalidade por causas externas segundo sexo e ano, São Luís, MA, 1980 a 1999.

Table 2 - Number of deaths and mortality coefficients due to external causes according to gender and year, São Luís, MA, 1980 to 1999.

\begin{tabular}{lcccccc}
\hline Ano & \multicolumn{2}{c}{ Masculino } & \multicolumn{2}{c}{ Feminino } & \multicolumn{2}{c}{ Total } \\
& Óbitos & Coef. & Óbitos & Coef. & Óbitos & Coef. \\
\hline 1980 & 211 & 100,6 & 76 & 31,7 & 287 & 63,9 \\
1981 & 284 & 130,0 & 82 & 32,8 & 366 & 78,1 \\
1982 & 329 & 143,5 & 81 & 30,8 & 410 & 83,4 \\
1983 & 333 & 138,7 & 71 & 25,8 & 404 & 78,4 \\
1984 & 305 & 121,6 & 68 & 23,7 & 374 & 69,5 \\
1985 & 303 & 115,8 & 92 & 30,7 & 395 & 70,3 \\
1986 & 347 & 127,4 & 95 & 30,4 & 442 & 75,6 \\
1987 & 339 & 119,8 & 95 & 29,3 & 434 & 71,5 \\
1988 & 398 & 135,7 & 100 & 29,7 & 498 & 79,1 \\
1989 & 353 & 116,4 & 100 & 28,8 & 453 & 69,6 \\
1990 & 371 & 118,5 & 79 & 22,0 & 450 & 67,0 \\
1991 & 370 & 114,1 & 107 & 28,8 & 478 & 68,6 \\
1992 & 362 & 109,3 & 62 & 16,3 & 424 & 59,6 \\
1993 & 347 & 100,9 & 76 & 19,3 & 424 & 57,4 \\
1994 & 320 & 91,8 & 75 & 18,7 & 396 & 52,9 \\
1995 & 412 & 116,6 & 92 & 22,7 & 508 & 66,9 \\
1996 & 443 & 122,2 & 97 & 23,2 & 542 & 69,4 \\
1997 & 422 & 113,4 & 100 & 23,3 & 524 & 65,3 \\
1998 & 422 & 110,9 & 91 & 20,7 & 513 & 62,6 \\
1999 & 360 & 92,6 & 87 & 19,4 & 447 & 53,4 \\
\hline
\end{tabular}

Coef.= Coeficiente de mortalidade por 100.000 habitantes

Em termos proporcionais, observou-se que a grande maioria dos óbitos ocorreu entre os homens. Em 1992, mais de 85\% das mortes verificaram-se entre os indivíduos do sexo masculino, enquanto o maior percentual entre as mulheres ocorreu em 1980, $26,5 \%$, quase quatro vezes menor. O maior coeficiente de mortalidade entre os homens foi observado no ano de 1982, com 143,5 por 100.000 habitantes. Entre as mulheres, o maior foi em 1981, com 32,8 por 100.000 habitantes. $\mathrm{O}$ coeficiente de mortalidade por causas externas no sexo masculino foi aproximadamente 4,5 vezes superior ao feminino, sendo de três a seis vezes maior ao longo da série histórica (Tabela 2). O coeficiente de mortalidade por causas externas diminuiu significantemente em ambos os sexos, sendo a redução mais acentuada no sexo feminino ( $2,5 \%$ ao ano) do que no masculino $(1,2 \%$ ao ano) (Tabela 3$)$.
Os maiores coeficientes de mortalidade por causas externas foram observados no grupo com 70 anos e mais, e os menores no grupo de 0 a 9 anos (Tabela 4). As faixas etárias de 0 a 9,30 a 39 e 40 a 49 anos mostraram tendência de queda extremamente significante $(\mathrm{p}<0,001)$, sendo a maior redução observada dos 0 aos 9 anos (3,5\%). Nas demais faixas, as variações observadas não foram significantes (Tabela 3).

Os acidentes de transporte iniciaram a série histórica com um coeficiente relativamente alto, de 25,4 por 100.000 habitantes. Já no ano seguinte alcançaram o seu maior coeficiente, 30,7 por 100.000 habitantes. Atingiram os seus menores coeficientes entre os anos de 1984 e 1985, aproximadamente 7 por 100.000 habitantes. A partir de então experimentaram novo crescimento até atingirem, no ano de $1988,26,5$ por 100.000 habitantes. Voltaram a declinar até o final do período, 
Tabela 3 - Análise de tendências da mortalidade por causas externas segundo sexo, idade e tipo, em São Luís, 1980 a 1997.

Table 3 - Analysis of mortality trends due to external causes according to gender, age and type, in São Luís, 1980 to 1997.

\begin{tabular}{|c|c|c|c|}
\hline Variáveis & Risco Relativo & Intervalo de Confiança de $95 \%$ & $P$ \\
\hline \multicolumn{4}{|l|}{ Sexo } \\
\hline Masculino & 0.988 & $0.984-0.993$ & $<0,001$ \\
\hline Feminino & 0.975 & $0.967-0.983$ & $<0,001$ \\
\hline \multicolumn{4}{|l|}{ Faixa etária (anos) } \\
\hline 0 a 9 & 0.965 & $0.954-0.976$ & $<0,001$ \\
\hline 10 a 19 & 0.996 & 0.987-1.005 & 0.404 \\
\hline 20 a 29 & 0.996 & $0.989-1.003$ & 0.263 \\
\hline 30 a 39 & 0.983 & $0.974-0.992$ & $<0,001$ \\
\hline 40 a 49 & 0.979 & $0.968-0.990$ & $<0,001$ \\
\hline 50 a 59 & 0.987 & $0.971-1.002$ & 0.090 \\
\hline 60 a 69 & 0.995 & $0.976-1.014$ & 0.594 \\
\hline$\geq 70$ & 0.999 & $0.982-1.016$ & 0.893 \\
\hline \multicolumn{4}{|l|}{ Tipo } \\
\hline Acidentes de transporte & 0.980 & $0.973-0.987$ & $<0,001$ \\
\hline Demais acidentes & 0.955 & $0.949-0.961$ & $<0,001$ \\
\hline Suicídios & 1.051 & $1.028-1.074$ & $<0,001$ \\
\hline Homicídios & 1.035 & $1.027-1.043$ & $<0,001$ \\
\hline Ignorados & 0.977 & $0.967-0.987$ & $<0,001$ \\
\hline Geral & 0.986 & $0.982-0.989$ & $<0,001$ \\
\hline
\end{tabular}

Tabela 4 - Número de óbitos e coeficientes de mortalidade por causas externas segundo faixa etária e ano, São Luís, MA, 1980 a 1999.

Table 4 - Number of deaths and mortality coefficients due to external causes according to age and year, São Luís, MA, 1980 to 1999

\begin{tabular}{|c|c|c|c|c|c|c|c|c|c|c|c|c|c|c|c|c|}
\hline \multirow[t]{2}{*}{ Ano } & \multicolumn{2}{|c|}{0 a 9} & \multicolumn{2}{|c|}{10 a 19} & \multicolumn{2}{|c|}{20 a 29} & \multicolumn{2}{|c|}{30 a 39} & \multicolumn{2}{|c|}{40 a 49} & \multicolumn{2}{|c|}{50 a 59} & \multicolumn{2}{|c|}{60 a 69} & \multicolumn{2}{|c|}{70 e mais } \\
\hline & Óbitos & Coef. & Óbitos & Coef. & Óbitos & Coef. & Óbitos & Coef. & Óbitos & Coef. & Óbitos & Coef. & Óbitos & Coef. & Óbitos & Coef. \\
\hline 1980 & 49 & 40,5 & 46 & 38,8 & 76 & 87,7 & 49 & 101,0 & 34 & 105,2 & 13 & 61,5 & 14 & 113,4 & 4 & 47,6 \\
\hline 1981 & 53 & 42,4 & 77 & 62,5 & 84 & 92,4 & 55 & 106,0 & 41 & 120,5 & 29 & 131,5 & 7 & 54,1 & 14 & 163,8 \\
\hline 1982 & 72 & 55,5 & 46 & 35,8 & 115 & 119,8 & 73 & 130,6 & 43 & 119,4 & 21 & 91,1 & 17 & 124,6 & 17 & 188,3 \\
\hline 1983 & 48 & 35,7 & 72 & 53,8 & 115 & 113,9 & 63 & 105,1 & 48 & 126,2 & 22 & 91,5 & 20 & 139,4 & 15 & 157,8 \\
\hline 1984 & 52 & 37,4 & 51 & 36,6 & 99 & 93,4 & 86 & 134,5 & 36 & 90,0 & 18 & 71,9 & 17 & 112,9 & 13 & 130,2 \\
\hline 1985 & 60 & 41,7 & 60 & 41,5 & 106 & 95,5 & 82 & 120,6 & 36 & 85,7 & 23 & 88,4 & 14 & 88,8 & 13 & 124,3 \\
\hline 1986 & 58 & 39,1 & 69 & 46,0 & 123 & 106,1 & 73 & 101,5 & 46 & 104,6 & 27 & 100,0 & 14 & 85,0 & 24 & 219,6 \\
\hline 1987 & 58 & 37,9 & 58 & 37,4 & 121 & 100,1 & 76 & 100,2 & 53 & 115,4 & 23 & 82,2 & 17 & 99,1 & 20 & 175,5 \\
\hline 1988 & 79 & 50,2 & 62 & 38,7 & 113 & 90,0 & 92 & 115,4 & 68 & 142 & 33 & 114,1 & 15 & 84,1 & 26 & 219,4 \\
\hline 1989 & 54 & 33,4 & 70 & 42,4 & 119 & 91,3 & 84 & 100,7 & 53 & 106,7 & 23 & 77,1 & 19 & 102,8 & 22 & 179,0 \\
\hline 1990 & 53 & 31,9 & 77 & 45,3 & 122 & 90,5 & 82 & & 45 & 87,4 & 16 & & 20 & 104,6 & 18 & 141,5 \\
\hline 1991 & 46 & 26,9 & 68 & 38,7 & 146 & 104,2 & 78 & 85,5 & 40 & 74 & 32 & 100,7 & 19 & 5,6 & 29 & 219,3 \\
\hline 1992 & 40 & 23,7 & 61 & 33,9 & 129 & & 73 & 76,7 & 46 & & 19 & & 23 & 112,0 & 20 & 142,3 \\
\hline 1993 & 44 & 24,3 & 74 & 39,7 & 124 & 83 & 63 & 65, & 45 & & 34 & 101,0 & 16 & & 5 & 107,0 \\
\hline 1994 & 40 & 21, & 7 & 40 & 99 & & 68 & & 35 & & 5 & & 17 & & 3 & 161,8 \\
\hline 1995 & 47 & 25 & & 45 & & & 90 & & 51 & & 26 & & 23 & 106,2 & 25 & 173,5 \\
\hline 1996 & 36 & 22,9 & 91 & 45,7 & 150 & 94,9 & 113 & 100,8 & 62 & 88,8 & 37 & & 18 & & 24 & 135,5 \\
\hline 1997 & 43 & 26,6 & 76 & 37,2 & 157 & 96,7 & 105 & 91,2 & 59 & 82,3 & 32 & 79,4 & 21 & 87,2 & 26 & 142,9 \\
\hline 1998 & 36 & 21,8 & 78 & 37,3 & 153 & 92,2 & 102 & 86,7 & 54 & 73,7 & 17 & 41,3 & 23 & 93,4 & 36 & 193,6 \\
\hline 1999 & 32 & 18,9 & 63 & 29,5 & 118 & 69,6 & 90 & 74,9 & 70 & 93,5 & 22 & 52,3 & 23 & 91,4 & 26 & 136,8 \\
\hline
\end{tabular}

Coef.= Coeficiente de mortalidade por 100.000 habitantes 
encerrando a série com 9,4 por 100.000 habitantes (Tabela 5). Os acidentes de transporte demonstraram tendência de queda de $2 \%$ ao ano, extremamente significante $(\mathrm{p}<0,001)$ (Tabela 3$)$.

Embora tenha iniciado a série com um coeficiente relativamente baixo, 8,2 por 100.000 habitantes, o grupo dos demais acidentes apresentou um rápido crescimento. Já no terceiro ano da série atingiu o maior coeficiente de mortalidade do período, 45,8 por 100.000 habitantes. Logo em seguida entrou em declínio, encerrando a série com 16,5 por 100.000 habitantes (Tabela 5). O decréscimo observado nos coeficientes de mortalidade deste grupo, de $4,5 \%$ ao ano, foi extremamente significante $(\mathrm{p}<0,001)$ (Tabela 3$)$. Nesse grupo, as causas mais freqüentes foram os afogamentos e as quedas acidentais.

Os homicídios apresentaram os seus menores coeficientes de mortalidade entre os anos de 1983 e 1985, com aproximadamente 3,5 por 100.000 habitantes. A partir de então, apresentaram rápido crescimento, até atingirem o seu maior coeficiente no ano de 1991 com 26,6 por 100.000 habitantes. Declinaram a seguir até encerrar o período com 11,9 por 100.000 habitantes, valor ligeiramente superior ao inicial, que foi 10,2 por 100.000 habitantes (Tabela 5). Observou-se tendência de aumento altamente significante, de $3,5 \%$ ao ano, da mortalidade por homicídios (p<0,001) (Tabela 3).

Os suicídios apresentaram durante todo o período coeficientes em torno de 2,0 por 100.000 habitantes. O maior coeficiente de mortalidade por suicídios, 4,1 por 100.000 habitantes, foi observado em 1996 (Tabela 5). Houve tendência de elevação extremamente significante da mortalidade por suicídio no período, de $5,1 \%$ ao ano $(\mathrm{p}<0,001)$ (Tabela3)

Tabela 5 - Número de óbitos e coeficientes de mortalidade por causas externas segundo tipo e ano, São Luís, MA, 1980 a 1999.

Table 5 - Number of deaths and mortality coefficients due to external causes according to type and year, São Luis, MA, 1980 to 1999

\begin{tabular}{|c|c|c|c|c|c|c|c|c|c|c|}
\hline \multirow[t]{2}{*}{ Ano } & \multicolumn{2}{|c|}{$\begin{array}{l}\text { Acidentes de } \\
\text { Transporte }\end{array}$} & \multicolumn{2}{|c|}{$\begin{array}{c}\text { Demais } \\
\text { Acidentes }\end{array}$} & \multicolumn{2}{|c|}{ Suicídios } & \multicolumn{2}{|c|}{ Homicídios } & \multicolumn{2}{|c|}{ Ignoradas } \\
\hline & Óbitos & Coef. & Óbitos & Coef. & Óbitos & Coef. & Óbitos & Coef. & Óbitos & Coef. \\
\hline 1980 & 114 & 25,4 & 37 & 8,2 & 10 & 2,2 & 46 & 10,2 & 80 & 17,8 \\
\hline 1981 & 144 & 30,7 & 135 & 28,8 & 11 & 2,3 & 72 & 15,4 & 4 & 0,9 \\
\hline 1982 & 92 & 18,7 & 225 & 45,8 & 0 & 0,0 & 53 & 10,8 & 40 & 8,1 \\
\hline 1983 & 46 & 8,9 & 211 & 41,0 & 7 & 1,4 & 15 & 2,9 & 125 & 24,3 \\
\hline 1984 & 41 & 7,6 & 194 & 36,0 & 5 & 0,9 & 23 & 4,3 & 111 & 20,6 \\
\hline 1985 & 40 & 7,1 & 237 & 42,2 & 4 & 0,7 & 25 & 4,5 & 89 & 15,8 \\
\hline 1986 & 84 & 14,4 & 209 & 35,8 & 12 & 2,1 & 69 & 11,8 & 68 & 11,6 \\
\hline 1987 & 119 & 19,6 & 172 & 28,3 & 8 & 1,3 & 91 & 15,0 & 44 & 7,2 \\
\hline 1988 & 167 & 26,5 & 178 & 28,3 & 15 & 2,4 & 118 & 18,7 & 20 & 3,2 \\
\hline 1989 & 157 & 24,1 & 121 & 18,6 & 9 & 1,4 & 161 & 24,7 & 5 & 0,8 \\
\hline 1990 & 146 & 21,7 & 125 & 18,6 & 12 & 1,8 & 161 & 24,0 & 6 & 0,9 \\
\hline 1991 & 149 & 21,4 & 117 & 16,8 & 22 & 3,2 & 185 & 26,6 & 5 & 0,7 \\
\hline 1992 & 157 & 22,1 & 94 & 13,2 & 12 & 1,7 & 156 & 21,9 & 5 & 0,7 \\
\hline 1993 & 135 & 18,3 & 124 & 16,8 & 18 & 2,4 & 127 & 17,2 & 20 & 2,7 \\
\hline 1994 & 125 & 16,7 & 102 & 13,6 & 15 & 2,0 & 139 & 18,6 & 15 & 2,0 \\
\hline 1995 & 147 & 19,4 & 142 & 18,7 & 17 & 2,2 & 184 & 24,2 & 18 & 2,4 \\
\hline 1996 & 137 & 17,5 & 169 & 21,6 & 32 & 4,1 & 166 & 21,3 & 38 & 4,9 \\
\hline 1997 & 103 & 12,8 & 162 & 20,2 & 25 & 3,1 & 160 & 20,0 & 74 & 9,2 \\
\hline 1998 & 65 & 7,9 & 124 & 15,1 & 19 & 2,3 & 123 & 15,0 & 182 & 22,2 \\
\hline 1999 & 79 & 9,4 & 138 & 16,5 & 24 & 2,9 & 100 & 11,9 & 106 & 12,7 \\
\hline
\end{tabular}

Coef.= Coeficiente de mortalidade por 100.000 habitantes 
O grupo dos ignorados iniciou a série histórica em 1980 com um coeficiente relativamente elevado, 17,8 por 100.000 habitantes, experimentando ligeira queda no ano seguinte. Voltou a crescer a partir do ano de 1982, para atingir, entre os anos de 1983 e 1985, coeficientes de aproximadamente 20 por 100.000 habitantes. Período este que correspondeu aos menores coeficientes de mortalidade por acidentes de transporte e homicídios. A partir de então, apresentou queda acentuada até 1992, quando voltou a crescer, apresentando pico de 22,2 por 100.000 habitantes em 1998 e encerrando o período com 12,7 por 100.000 habitantes (Tabela 5). Observou-se tendência de queda altamente significante para este grupo, de $2,3 \%$ ao ano $(\mathrm{p}<0,001)$ (Tabela 3$)$.

\section{Discussão}

O coeficiente de mortalidade por causas externas diminuiu de maneira significante, tanto no sexo masculino como no feminino. Reduções também foram observadas nas faixas etárias de 0 a 9, 30 a 39 e de 40 a 49 anos. Observou-se aumento da mortalidade por homicídios e suicídios. Houve redução na mortalidade por acidentes de transporte, demais acidentes e por causas externas do tipo ignorado, indicando melhoria da qualidade da informação ao longo do período.

A tendência de queda da mortalidade por causas externas em São Luís, de 28\% de 1980 a 1999, está de acordo com observações mais recentes feitas por Yunes \& Zubarew ${ }^{3}$ (1999), que concluíram que a mortalidade por essas causas apresentou tendência de queda na região das Américas e com dados do município de São Paulo ${ }^{15}$ e do Brasil como um todo $^{2}$. Ao longo da série histórica, esta queda se deveu mais à redução no sexo feminino $(50 \%)$ do que no masculino (24\%) e decresceu mais dos 0 aos 9 anos (70\%) do que dos 40 aos 49 (42\%) ou dos 30 aos 39 anos (34\%). A redução da mortalidade dos 0 aos 9 anos concorda com observações recentes feitas em Recife ${ }^{16}$.

No Brasil, Mello Jorge et al. ${ }^{1}$ (1997), encontraram entre 1977 e 1994 um aumento de aproximadamente $17 \%$ na mortalidade por acidentes de trânsito. Entretanto, em São Luís foi observada queda extremamente significante, de $40 \%$ entre 1980 e 1999, o que coincide com observações mais recentes feitas para o Brasil como um todo ${ }^{2}$ e para o município de São Paulo, onde, a partir da década de 80 os acidentes de trânsito começaram a apresentar decréscimo, tendo diminuído 55,7\% de 1975 a $1999^{15}$.

A queda significante observada na mortalidade para os demais acidentes foi a de maior magnitude, de $90 \%$ de 1980 a 1999, sendo este item o que mais contribuiu para a queda observada na mortalidade pelas causas externas. A tendência de queda da mortalidade por demais acidentes não foi observada em São Paulo, onde este item, apesar das flutuações, apresentou-se mais ou menos estável ${ }^{15}$. A faixa etária onde ocorreu a maior proporção de óbitos por demais acidentes foi de 0 a 9 anos. As causas mais freqüentes neste grupo por ordem decrescente foram os acidentes não especificados, afogamentos, acidentes por corrente elétrica e as quedas acidentais. É possível que muitos destes óbitos por demais acidentes estejam relacionados à construção civil, sendo necessárias novas investigações a este respeito.

Para o Brasil, Mello Jorge et al. ${ }^{1}$ (1997) encontraram aumento de $168 \%$ da mortalidade por homicídios entre 1977 e 1994. Em São Paulo, a mortalidade por homicídios aumentou 906,8\%, de 1975 a $1999^{15}$. Em São Luís, foi encontrado aumento bem menor, de $70 \%$, entre 1980 e 1999. Aumento expressivo da mortalidade por homicídios também foi detectada em Salvador ${ }^{8}$. O aumento da mortalidade por homicídios deveu-se, quase exclusivamente, às mortes no sexo masculino. Apesar de São Luís ser uma cidade de menor renda per capita do que São Paulo, tem mortalidade por homicídios bastante inferior à de São Paulo. Estes dados sugerem que a mortalidade por homicídios está muito mais ligada ao crime organizado e a atividades ligadas ao tráfico de drogas do que à pobreza.

Os suicídios não se mostraram importantes em freqüência, apresentando patama- 
res muito baixos, se comparados com os do Brasil (1994), 3,9 por 100.000 habitantes, e com outros países como o Japão (1992) com 16,9 por 100.000 habitantes; os Estados Unidos, 12,4 por 100.000 habitantes, em 1990; e a França, 20,2 por 100.000 habitantes, em $1991^{1}$. Apesar de sua pequena magnitude, apresentou tendência de aumento significante, de $102 \%$ ao longo da série, o que discorda dos dados deSão Paulo, onde este item apresentou redução de 65,1\% de 1975 a $1999^{15}$.

Nos anos de 1983 a 1985, e de 1998 a 1999, foram observados os maiores percentuais de morte por causas externas do tipo "ignorado". Isto correspondeu exatamente aos menores percentuais e coeficientes de mortalidade verificados para os acidentes de transporte e homicídios, nesses anos. Tal fato sugere que esses altos índices de "ignorados" sejam devidos, na realidade, a casos de acidentes de transporte e homicídios não devidamente esclarecidos. Rotulados como "ignorados", elevaram os percentuais destes em detrimento daqueles. Para verificar se este provável erro de classificação poderia levar a conclusões diferentes, foram rodados modelos de regressão excluindo-se estes anos. Os resultados foram aproximadamente os mesmos, o que demonstra que este erro não interferiu nos resultados aqui apontados. Outra limitação do presente estudo foi o pequeno número de óbitos ocorridos em algumas faixas etárias, o que reduziu o poder do estudo para identificar tendências significantes.

A diminuição acentuada do número de óbitos por causas externas de causa ignorada pode ter sido devida à metodologia adotada pela então Secretaria de Saúde. Este órgão reclassificava as causas dos óbitos por causas externas baseado em informações adicionais obtidas em notícias de jornais ${ }^{1}$. Entre 1980 e 1999, os percentuais de ignorados declinaram 46\% em São Luís, enquanto para o Brasil em geral, de 1977 a 1994, permaneceram praticamente estáveis. Para o Brasil, esse tipo de causas externas tem-se mantido ao redor de $15 \%^{1}$. Em São Luís, o valor foi $12 \%$.
A razão desse elevado número de "ignorados" deve-se na realidade ao incompleto preenchimento da Declaração de Óbito por parte dos médicos legistas. Estes, com receio de se envolverem com a justiça e/ou a polícia, registram na declaração a "causa médica” da morte, ou seja, a natureza do traumatismo ou lesão que levou ao óbito, sem qualquer referência à verdadeira causa básica da morte, definida pela Organização Mundial de Saúde como o "tipo de acidente ou violência que ocasionaram as lesões que levaram à morte".

Uma das limitações do presente trabalho é que os dados de mortalidade do Estado do Maranhão estão entre os de piores cobertura e qualidade do país ${ }^{2}$. Desta forma, as conclusões do presente estudo devem ser interpretadas tendo-se em mente esta limitação.

Diante desse problema, sugere-se o empenho dos órgãos de saúde junto ao Instituto Médico Legal, no sentido de que os médicos legistas sejam orientados sobre a importância da Declaração de Óbito - dando a informação completa sobre cada caso (por exemplo, politraumatismo conseqüente a atropelamento por automóvel; traumatismo crânio-encefálico devido a homicídio por arma de fogo). É importante que os legistas anotem na Declaração de Óbito a fonte de onde foi retirada a informação ali transcrita (Boletim de Ocorrência Policial), para que não venham a ter envolvimento futuro com autoridades policiais ou judiciais ${ }^{17}$. Somente dessa maneira será possível a obtenção de dados mais precisos quanto às causas externas, a fim de que, com melhores estatísticas oficiais, programas efetivos de prevenção dessas mortes venham a ser realizados.

\section{Agradecimentos}

Este trabalho foi baseado na Dissertação de Mestrado em Saúde e Ambiente de José Ribamar Moraes, apresentada à Universidade Federal do Maranhão em 2001. Agradecemos as valiosas sugestões do professor Jairnílson Silva Paim, do Instituto de Saúde Coletiva da Universidade Federal da Bahia. 


\section{Referências}

1. Mello Jorge MHP, Gawryszewski VP, Latorre MRD. Análise dos dados de mortalidade. Rev Saúde Pública 1997; 31: 5-24.

2. Mello Jorge MHP, Gotlieb SLD, Laurenti R. A saúde no Brasil: análise do período 1996 a 1999. Brasília: Organização Pan-Americana da Saúde; 2001.

3. Yunes J, Zubarew T. Mortalidad por causas violentas en adolescentes y jóvenes: un desafio para la región de las Américas. Rev Bras Epidemiol 1999; 2: 102-71.

4. Mello Jorge MHP, Latorre MRD. Acidentes de Trânsito no Brasil: Dados e Tendências. Cad Saúde Pública 1994; 10: 7-18.

5. Yunes J. Mortalidad por causas violentas en la región de las Américas. Bol Oficina Sanit Panam 1993; 114: 302-316.

6. Souza ER, Minayo MCS. O impacto da violência social na saúde pública do Brasil: década de 80. In: Minayo MCS, org. Os muitos Brasis: saúde e população na década de 80. São Paulo/Rio de Janeiro: Hucitec/ Abrasco; 1995. p. 87-116.

7. Souza ER. Homicídios no Brasil: o grande vilão da Saúde Pública na década de 80. Cad Saúde Pública 1994; 10: 45-60.

8. Freitas ED, Paim JS, Silva LMV, Costa MCN. Evolução e distribuição espacial da mortalidade por causas externas em Salvador, Bahia, Brasil. Cad Saúde Pública 2000; 16: 1059-70.

9. Macedo AC, Paim JS, Silva LMV, Costa MCN. Violência e desigualdade social: mortalidade por homicídios e condições de vida em Salvador, Brasil. Rev Saúde Pública 2001; 35: 515-22.
10. DATASUS. População residente por faixa etária segundo ano. São Luís; 2001. <http:// www.datasus.gov.br/cgi/tabcgi.exe?ibge/popma.def>

11. Organização Mundial de Saúde. Classificação Estatística Internacional de Doenças, Lesões e Causas de Óbitos. Revisão 1975. São Paulo: Centro Colaborador da OMS para Classificação das Doenças em Português/EDUSP; 1978. v 1.

12. Organização Mundial de Saúde. Classificação Internacional de Doenças e Problemas Relacionados à Saúde: $10^{a}$ revisão. São Paulo: EDUSP; 1995. v 1.

13. Woodward M. Epidemiology: study design and data analysis. Boca Raton: Chapman \& Hall/CRC; 1999.

14. Stata Corporation, Stata Statistical Software: Release 6. College Station, Texas: Stata Corporation; 1999.

15. Gawryszewski VP, Mello Jorge MHP. Mortalidade violenta no município de São Paulo nos últimos 40 anos. Rev Bras Epidemiol 2000; 3: 50-69.

16. Barros MDA, Ximenes R, Lima MLC. Mortalidade por causas externas em crianças e adolescentes: tendências de 1979 a 1995. Rev Saúde Pública 2001; 35: 142-9.

17. Mello Jorge MHP. Situação atual das estatísticas oficiais relativas à mortalidade por causas externas. Rev Saúde Pública 1990; 24: 217-23.

Recebido em: 31/07/2002 Versão final reapresentada em: 22/08/2002 Aprovação em: 10/06/2003 